\title{
WASP-147b, 160Bb, 164b and 165b: two hot Saturns and two Jupiters, including two planets with metal-rich hosts
}

\author{
M. Lendl ${ }^{1,2 \star}$, D.R. Anderson ${ }^{3}$, A. Bonfanti ${ }^{4}$, F. Bouchy ${ }^{2}$, A. Burdanov ${ }^{4}$, \\ A. Collier Cameron ${ }^{5}$, L. Delrez ${ }^{6,4}$, M. Gillon ${ }^{4}$, C. Hellier ${ }^{3}$, E. Jehin ${ }^{4}$, \\ P.F.L. Maxted ${ }^{3}$, L. Dyregaard Nielsen ${ }^{2}$, F. Pepe ${ }^{2}$, D. Pollacco ${ }^{7}$, D. Queloz ${ }^{2,6}$, \\ D. Ségransan ${ }^{2}$, J. Southworth ${ }^{3}$, B. Smalley ${ }^{3}$, S. Thompson ${ }^{6}$, O. Turner ${ }^{2}$, \\ A.H.M.J. Triaud ${ }^{8}$, S. Udry ${ }^{2}$, and R.G. West ${ }^{7}$ \\ ${ }^{1}$ Space Research Institute, Austrian Academy of Sciences, Schmiedlstr. 6, 8042 Graz, Austria \\ ${ }^{2}$ Observatoire de Genève, Université de Genève, Chemin des maillettes 51, 1290 Sauverny, Switzerland \\ ${ }^{3}$ Astrophysics Group, Keele University, Staffordshire, ST5 5BG, UK \\ ${ }^{4}$ Space sciences, Technologies and Astrophysics Research (STAR) Institute, Université de Liège, Allée du 6 Août 17, 4000 Liège, Belgium \\ ${ }^{5}$ SUPA, School of Physics and Astronomy, University of St. Andrews, North Haugh, Fife, KY16 9SS, UK \\ ${ }^{6}$ Cavendish Laboratory, J J Thomson Avenue, Cambridge, CB3 OHE, UK \\ ${ }^{7}$ Department of Physics, University of Warwick, Gibbet Hill Road, Coventry CV4 7AL, UK \\ ${ }^{8}$ School of Physics 83 Astronomy, University of Birmingham, Edgbaston, Birmingham B15 2TT, UK
}

Accepted XXX. Received YYY; in original form ZZZ

\begin{abstract}
We report the discovery of four transiting hot Jupiters, WASP-147, WASP-160B, WASP-164 and WASP-165 from the WASP survey. WASP-147b is a near Saturn-mass $\left(M_{P}=0.28 M_{J}\right)$ object with a radius of $1.11 R_{J}$ orbiting a G4 star with a period of $4.6 \mathrm{~d}$. WASP-160Bb has a mass and radius $\left(M_{p}=0.28 M_{J}, R_{p}=1.09 R_{J}\right)$ near-identical to WASP-147b, but is less irradiated, orbiting a metal-rich $\left([\mathrm{Fe} / \mathrm{H}]_{*}=0.27\right) \mathrm{K} 0$ star with a period of $3.8 \mathrm{~d}$. WASP-160B is part of a near equal-mass visual binary with an on-sky separation of 28.5 arcsec. WASP-164b is a more massive $\left(M_{P}=2.13 M_{J}\right.$, $\left.R_{p}=1.13 R_{J}\right)$ hot Jupiter, orbiting a G2 star on a close-in $(P=1.8 \mathrm{~d})$, but tidally stable orbit. WASP-165b is a classical $\left(M_{p}=0.66 M_{J}, R_{P}=1.26 R_{J}\right)$ hot Jupiter in a $3.5 \mathrm{~d}$ period orbit around a metal-rich $\left([\mathrm{Fe} / \mathrm{H}]_{*}=0.33\right)$ star. WASP-147b and WASP$160 \mathrm{Bb}$ are promising targets for atmospheric characterization through transmission spectroscopy, while WASP-164b presents a good target for emission spectroscopy.
\end{abstract}

Key words: Planetary Systems - planets and satellites: detection - (stars:) planetary systems

\section{INTRODUCTION}

Transiting exoplanets are invaluable objects for study. Not only are both, their masses and radii known, but also their transiting configuration opens up a wide range of characterization avenues. We may study the atmospheres of these objects through their transmission and emission spectra (Seager \& Sasselov 2000; Charbonneau et al. 2002, 2005), but also measure their orbital alignment (Queloz et al. 2000); see Triaud (2017) for a summary. While over 2700 transiting planets are known to date ${ }^{1}$, only a fraction of these objects

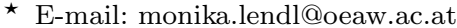

1 according to exoplanets.eu, queried on 12 Apr 2018.
}

are suitable for detailed characterization, as this requires the planet host to be bright and the star/planet size ratio to be favorable.

Ground based transit surveys (e.g. WASP, Pollacco et al. 2006; HAT, Bakos et al. 2004; KELT, Pepper et al. 2007; and MASCARA,Talens et al. 2017) use small-aperture instrumentation to monitor vast numbers of bright stars across nearly the entire sky, sensitive to the $\sim 1 \%$ dips created by transits of close-in giant planets. These hot Jupiters are prime targets for further characterization thanks to their large radii, frequent transits and extended atmospheres. Indeed, ground-based transit surveys have provided some of the most intensely studied planets to date (e.g. WASP-12b, 
Hebb et al. 2009; WASP-43b, Hellier et al. 2011 and HATP-11b, Bakos et al. 2010).

In this paper, we report the discovery of four additional close-in transiting gas giants by WASP-South, the two Saturn-mass planets WASP-147b and WASP-160Bb, and the two hot Jupiters WASP-164b and WASP-165b. We discuss the observations leading to these discoveries in Section 2 , describe their host stars in Section 3 and discuss the individual planetary systems and their place among the known planet population in Section 4 before concluding in Section 5.

\section{OBSERVATIONS}

WASP-147 (2MASS 23564597-2209113), WASP-160B (2MASS 05504305-2737233), WASP-164 (2MASS 225929626026519) and WASP-165 (2MASS 23501932-1704392) were monitored with the WASP-South facility throughout several years between 2006 and 2013. In the case of WASP-160B, the target flux was blended with that of another object (2MASS 05504470-2737050, revealing to be physically associated, see below) in the WASP aperture. The WASP-South instrument consists of an array of 8 cameras equipped with $200 \mathrm{~mm} \mathrm{f} / 8$ Canon lenses on a single mount and is located at SAAO (South Africa). For details on observing strategy, data reduction and target selection, please refer to Pollacco et al. (2006) and Collier Cameron et al. (2007). Using the algorithms described by Collier Cameron et al. (2006), we identified periodic flux drops compatible with transits of close-in giant planets in the light curves of these four objects. We thus triggered spectroscopic and photometric follow-up observations to determine the nature of the observed dimmings.

\subsection{Follow-up spectroscopy}

We obtained spectroscopic observations of all four objects using the CORALIE echelle spectrograph at the $1.2 \mathrm{~m}$ EulerSwiss telescope at La Silla. From the spectra, we computed radial velocities (RVs) using the weighted cross-correlation method (Baranne et al. 1996; Pepe et al. 2002). In 2014, the CORALIE spectrograph was upgraded by replacing circular with octagonal fibers, leading to a shift in RV zero point between observations obtained before and after the exchange. WASP-160B, 164 and 165 were observed only after the upgrade, resulting in a single homogeneous set of RVs for each object. WASP-147 was observed before and after the upgrade, making it necessary to include these observations as two separate data sets in our analysis. For each of the four objects in question, RV variations confirmed the presence of a planet orbiting at the period of the observed transits (see Figs. 1 - 4). The individual RV measurements are listed in Tables $1-4$. To exclude stellar activity as the origin of the observed RV variations, we verified that bisector spans and RVs are uncorrelated (Queloz et al. 2001; Pearson coefficients are $-0.19 \pm 0.16,-0.16 \pm 0.19,-0.24 \pm 0.22$ and $0.06 \pm 0.22$ for WASP-147, 160B, 164 and 165, respectively). This is illustrated in Fig. 5, where we plot bisector spans against RVs. As both stellar components of the WASP-160AB system fell into the same WASP-South aperture, we could not a-priori exclude either of them as the origin of the observed transits.
We thus obtained several spectra of WASP-160A, showing no evidence of any large-amplitude RV variability (see Fig. $6)$.

\subsection{Follow-up photometry}

We obtained several high-precision transit light curves for each of our targets to obtain an improved measurement of the transit shape and depth. The facilities we used for this purpose were EulerCam at the $1.2 \mathrm{~m}$ Euler-Swiss telescope (Lendl et al. 2012), the 0.6m TRAPPIST-South telescope (Gillon et al. 2011; Jehin et al. 2011), and the SAAO 1.0m telescope. In all cases, we extracted light curves of the transit events using relative aperture photometry, while iteratively selecting reference stars and aperture sizes to minimize the final light curve RMS. Having an on-sky separation of $28.478948 \pm 2.5 \times 10^{-5}$ arcsec, both stellar components of the WASP-160 system were well-separated in these observations, confirming the fainter star, WASP-160B as the origin of the transit feature. Details on all photometric follow-up observations are listed in Table 5 . The resulting light curves are shown in Figs. 1-4.

\section{STELLAR PARAMETERS}

\subsection{Spectral analysis}

The individual CORALIE spectra for each star were coadded in order to provide a spectrum for analysis. Using methods similar to those described by Doyle et al. (2013), for each star we determined the effective temperature $\left(T_{\text {eff }}\right)$, surface gravity $(\log g)$, stellar metallicity $([\mathrm{Fe} / \mathrm{H}])$, and projected stellar rotational velocity $\left(v_{\text {rot }} \sin i_{*}\right)$. In determining $v_{\text {rot }} \sin i_{*}$ we assumed a macroturbulent velocity using the calibration given by (Doyle et al. 2014). For WASP-160B and WASP-165 the $v_{\text {rot }} \sin i_{*}$ values are consistent with zero and that of WASP-147 can be considered an upper limit, as it is close to the resolution limit of the CORALIE spectrograph. If, however, a zero macroturbulent velocity is used, we obtain $v_{\text {rot }} \sin i_{*}$ values of $3.1 \pm 0.5,0.7 \pm 0.6$ and $2.8 \pm 0.6 \mathrm{~km} \mathrm{~s}^{-1}$ for WASP-147, WASP-160B and WASP-165, respectively.

The parameters for WASP-164 are relatively poorly determined, as the signal-to-noise ratio of the merged spectrum

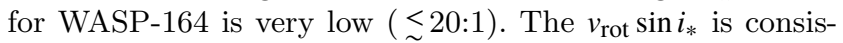
tent with zero, but very poorly determined and the Lithium $670.8 \mathrm{~nm}$ line might be present, but we cannot be sure due to the quality of the spectrum.

\subsection{Rotation periods}

The WASP light curves of WASP-164 show a quasi-periodic modulation with an amplitude of about 0.6 per cent and a period of about 18 days. We assume this is due to the combination of the star's rotation and magnetic activity, i.e., star spots. We used the sine-wave fitting method described in Maxted et al. (2011) to refine this estimate of the amplitude and period of the modulation. Variability due to star spots is not expected to be coherent on long timescales as a consequence of the finite lifetime of star-spots and differential rotation in the photosphere so we analyzed each season of data for WASP-164 separately. We also analyze the data 
Table 1. RV measurements of WASP-147. Only the first five entries are shown; the full table is available in the online version.

\begin{tabular}{ccccc}
\hline HJD-2450000 & RV $\left[\mathrm{km} \mathrm{s}^{-1}\right]$ & error $\left[\mathrm{km} \mathrm{s}^{-1}\right]$ & bisector $\left[\mathrm{km} \mathrm{s}^{-1}\right]$ & note \\
\hline 6135.904214 & -1.70365 & 0.02277 & -0.01228 & pre-upgrade \\
6137.920709 & -1.62092 & 0.02408 & -0.01027 & pre-upgrade \\
6157.712465 & -1.67809 & 0.03085 & 0.01114 & pre-upgrade \\
6158.661748 & -1.65414 & 0.01670 & 0.01027 & pre-upgrade \\
6508.712267 & -1.65458 & 0.02119 & 0.00580 & pre-upgrade \\
\hline
\end{tabular}

Table 2. RV measurements of WASP-160B. Only the first five entries are shown; the full table is available in the online version.

\begin{tabular}{cccc}
\hline HJD-2450000 & RV $\left[\mathrm{km} \mathrm{s}^{-1}\right]$ & error $\left[\mathrm{km} \mathrm{s}^{-1}\right]$ & bisector $\left[\mathrm{km} \mathrm{s}^{-1}\right]$ \\
\hline 7011.653767 & -6.16374 & 0.02709 & 0.03897 \\
7016.726245 & -6.12609 & 0.03159 & -0.07226 \\
7019.639217 & -6.15020 & 0.04051 & -0.03465 \\
7037.692732 & -6.20130 & 0.03296 & -0.01686 \\
7038.718708 & -6.11831 & 0.03588 & -0.04539 \\
\hline
\end{tabular}

Table 3. RV measurements of WASP-164. Only the first five entries are shown; the full table is available in the online version.

\begin{tabular}{cccc}
\hline HJD-2450000 & RV $\left[\mathrm{km} \mathrm{s}^{-1}\right]$ & error $\left[\mathrm{km} \mathrm{s}^{-1}\right]$ & bisector $\left[\mathrm{km} \mathrm{s}^{-1}\right]$ \\
\hline 7185.833271 & 12.66344 & 0.06016 & 0.00090 \\
7191.863118 & 11.92547 & 0.07861 & 0.18480 \\
7192.836522 & 12.62296 & 0.04957 & -0.08816 \\
7193.822033 & 11.95951 & 0.04570 & 0.05691 \\
7200.871113 & 11.97277 & 0.04621 & 0.08865 \\
\hline
\end{tabular}

Table 4. RV measurements of WASP-165. Only the first five entries are shown; the full table is available in the online version.

\begin{tabular}{cccc}
\hline HJD-2450000 & RV $\left[\mathrm{km} \mathrm{s}^{-1}\right]$ & error $\left[\mathrm{km} \mathrm{s}^{-1}\right]$ & bisector $\left[\mathrm{km} \mathrm{s}^{-1}\right]$ \\
\hline 7014.541384 & 25.74712 & 0.02309 & 0.06029 \\
7016.541690 & 25.56122 & 0.02932 & 0.03612 \\
7178.889363 & 25.65436 & 0.05627 & 0.08364 \\
7187.897899 & 25.70129 & 0.03835 & 0.05943 \\
7190.895590 & 25.66115 & 0.04947 & -0.00215 \\
\hline
\end{tabular}

from each camera used to observe WASP-164 separately so that we can assess the reliability of the results. We removed the transit signal from the data prior to calculating the periodograms by subtracting a simple transit model from the light curve. We calculated periodograms over 8192 uniformly spaced frequencies from 0 to 1.5 cycles/day. The false alarm probability (FAP) is calculated using a boot-strap Monte Carlo method also described in Maxted et al. (2011). The results are given in Table 7 and the periodograms and light curves for are shown in Fig. 7. There is a clear signal near 17.8 days in 4 out of 5 data sets, from which we obtain a value for the rotation period of $P_{\text {rot }}=17.81 \pm 0.03$ d. This rotation period together with our estimate the stellar radius implies a value of $V_{\text {rot }} \sin I=2.6 \pm 0.2 \mathrm{~km} \mathrm{~s}^{-1}$, assuming that the rotation axis of the star is approximately aligned with the orbital axis of the planet. This is consistent with the low value for $v_{\text {rot }} \sin i$ we obtain from our analysis of the spectroscopy of WASP-164. We used a least-squares fit of a sinusoidal function and its first harmonic to model the rotational modulation in the light curves for each camera and season with the rotation period fixed at $P_{\text {rot }}=17.81 \mathrm{~d}$.

For WASP-147, WASP-160B and WASP-165, a similar analysis leads to upper limits of 1.2 millimagnitudes, 2.9 millimagnitudes and 1.2 millimagnitudes with 95 per cent confidence for the amplitude of any sinusoidal signal over the same frequency range, respectively.

\subsection{Stellar evolution modeling}

In order to estimate stellar parameters, we considered $T_{\text {eff }}$, $[\mathrm{Fe} / \mathrm{H}], \log g$ and $v \sin i$ inferred from spectral analysis (see Sec. 3.1), the mean stellar density $\rho_{\star}$ inferred from the transit light curve (see Sec. 4.1) and magnitude $G$, color index $G_{B P}-G_{R P}$ and distance $d$ reported by Gaia DR2 (Gaia Collaboration et al. 2018). We analyzed WASP-147 and WASP160B using the set $G, G_{B P}-G_{R P}, d,[\mathrm{Fe} / \mathrm{H}], \log g, \rho_{\star}$ and $v \sin i$ as input parameters. For WASP-164 and WASP-165, we adopted the same input set, but replaced the color index by the spectroscopic temperature as it is more precisely known than that inferred from the Gaia colors. An extinction coefficient of $A v=0$ was assumed in this analysis. We recovered the main stellar parameters such as age, mass and radius according to theoretical models. We considered the grids of evolutionary tracks and isochrones computed by PARSEC $^{2}$ (version 1.2S; see Bressan et al. (2012); Chen et al. (2014) and references therein).

The interpolation of the input data in the theoretical grids to retrieve the output parameters has been done according to the isochrone placement technique described in Bonfanti et al. (2015, 2016). Here we briefly recall that the algorithm makes a comparison between observations and theoretical isochrones and select those theoretical data which match the observations best. In particular, for each star:

- multiple grids of isochrones spanning the input metallicity range $[[\mathrm{Fe} / \mathrm{H}]-\Delta[\mathrm{Fe} / \mathrm{H}] ;[\mathrm{Fe} / \mathrm{H}]+\Delta[\mathrm{Fe} / \mathrm{H}]]$ have been loaded;

- isochrones have been filtered through a 2-dimensional Gaussian window function whose $\sigma_{1}=\Delta T_{\text {eff }}, \sigma_{2}=\Delta \log L$;

- isochrones have been weighted evaluating the stellar evolutionary speed in the HR diagram and considering the similarity between theoretical and observational parameters;

- the gyrochronological relation by Barnes (2010) has been used to set a conservative age lower limit to discard unlikely very young isochrones;

2 Padova and Trieste Stellar Evolutionary Code. http://stev.oapd.inaf.it/cgi-bin/cmd 
Table 5. Summary of photometric follow-up observations together with the preferred baseline model, noise correction factors and the light curves' RMS per 5 minute bin. The notation of the baseline models, $p\left(j^{i}\right)$, refers to a polynomial of degree i in parameter j. Filter ranges: $\lambda_{\text {blue-block }}>500 \mathrm{~nm}, \lambda_{N G T S}=[500-900] \mathrm{nm}$, Wheatley et al. (2018).

\begin{tabular}{|c|c|c|c|c|c|c|}
\hline Date & Instrument & Filter & Baseline & $\beta_{r}$ & $\beta_{w}$ & $\mathrm{RMS}_{5 \min }[\mathrm{ppm}]$ \\
\hline \multicolumn{7}{|l|}{ WASP-147 } \\
\hline 05 Nov 2014 & TRAPPIST-South & $\mathrm{V}$ & $p\left(t^{2}\right)$ & 1.22 & 1.22 & 1419 \\
\hline 11 Nov 2013 & EulerCam & r' & $p\left(t^{2}\right)+p\left(F W H M^{1}\right)$ & 2.42 & 1.64 & 1020 \\
\hline 19 Oct 2013 & TRAPPIST-South & blue-block & $p\left(t^{2}\right)$ & 1.33 & 0.98 & 1682 \\
\hline 26 Sep 2013 & TRAPPIST-South & blue-block & $p\left(t^{2}\right)$ & 2.02 & 0.81 & 900 \\
\hline \multicolumn{7}{|l|}{ WASP-160B } \\
\hline 16 Dec 2014 & TRAPPIST-South & $\mathrm{I}+\mathrm{z}^{\prime}$ & $p\left(t^{2}\right)$ & 1.16 & 0.99 & 1091 \\
\hline 26 Dec 2015 & EulerCam & r' & $p\left(t^{2}\right)$ & 1.50 & 1.06 & 469 \\
\hline 02 Jan 2017 & EulerCam & r' & $p\left(t^{2}\right)$ & 1.92 & 1.08 & 628 \\
\hline \multicolumn{7}{|l|}{ WASP-164 } \\
\hline 29 Jun 2015 & TRAPPIST-South & blue-block & $p\left(t^{2}\right)$ & 1.58 & 0.89 & 1070 \\
\hline $31 \mathrm{Jul} 2015$ & EulerCam & r' & $p\left(t^{2}\right)+p\left(s k y^{1}\right)$ & 1.21 & 1.52 & 1103 \\
\hline 16 Aug 2015 & EulerCam & r' & $p\left(t^{2}\right)$ & 1.00 & 1.45 & 616 \\
\hline 25 Aug 2015 & EulerCam & r' & $p\left(t^{2}\right)+p\left(x y^{1}\right)$ & 1.09 & 1.31 & 503 \\
\hline 10 Sep 2016 & TRAPPIST-South & $\mathrm{I}+\mathrm{z}$ & $p\left(t^{2}\right)+p\left(F W H M^{2}\right)+p\left(x y^{1}\right)$ & 1.01 & 2.81 & 1301 \\
\hline 16 Oct 2016 & SAAO 1m & $\mathrm{R}$ & $p\left(t^{2}\right)$ & 1.61 & 0.85 & 1302 \\
\hline \multicolumn{7}{|l|}{ WASP-165 } \\
\hline 10 Mar 2015 & EulerCam & $N G T S$ & $p\left(t^{2}\right)+p\left(F W H M^{1}\right)$ & 1.52 & 1.60 & 623 \\
\hline 17 Sep 2016 & EulerCam & $N G T S$ & $p\left(t^{2}\right)+p\left(F W H M^{1}\right)$ & 1.32 & 1.46 & 626 \\
\hline 24 Sep 2016 & EulerCam & $N G T S$ & $p\left(t^{2}\right)+p\left(F W H M^{1}\right)$ & 1.98 & 1.41 & 559 \\
\hline
\end{tabular}

- element diffusion has been taken into account.

Uncertainties given by the code are simply internal, i.e. they are related to the interpolation scheme in use. Realistic uncertainties to be attributed to stellar parameters should take also theoretical model uncertainties into account. By comparing the results with two independent evolutionary models (namely PARSEC and CLES ${ }^{3}$, Scuflaire et al. 2008), we find that systematics due to models can be estimated to be $\sim 2 \%$. In addition, helium content $Y$ influences theoretical models, but its quantity cannot be estimated from spectroscopy (at least in the case of solar-like stars). Given the uncertainty on $Y$, a further $\sim 5 \%$ should be added to the error budget. Fig. 8 shows the placement of the planet hosts in the HR diagram.

We also used the open source software BAGEMASs ${ }^{4}$ to calculate the posterior mass distribution for each star using the Bayesian method described by Maxted et al. (2015). The models used in BAGEMASs were calculated using the GARSTEC stellar evolution code (Weiss \& Schlattl 2008) using as input the spectroscopically-derived $T_{\text {eff }}$ and $[\mathrm{Fe} / \mathrm{H}]$ as well as the transit-derived $\rho_{*}$ and orbital Period. The mass and age of the stars found are shown in Table 8. They are in excellent agreement with the values derived above for WASP-147, 164 and 165. For WASP-160B, BAGEMASS favors a slightly younger age and higher mass. This is due to the input selection of BAGEMASs that includes the spectroscopically-determined stellar effective temperature instead of the Gaia color index.

3 Code Liégeois d'Evolution Stellaire

${ }^{4}$ http://sourceforge.net/projects/bagemass

\subsection{The WASP-160 binary}

Due to the low resolution of the WASP instrument, photometry of WASP-160B was blended with a second, slightly brighter, source. While exploratory RV observations and transit follow-up quickly identified the origin of the transit the to be the fainter star, we found that both objects possess near-identical systemic RVs, pointing towards them being physically associated. This is confirmed by consistent Gaia proper motion and parallax values for both objects. To derive the properties of WASP-160A, we retrieved the stellar properties from a spectral analysis and stellar evolution models as described in Section 3.1 and 3.3. For the stellar evolution models, we used Gaia values for $G, G_{B P}-G_{R P}$ and $d$, and results from our spectroscopic analysis for $[\mathrm{Fe} / \mathrm{H}]$, $\log g$, and $v \sin i$ as inputs. As we have not detected any transiting planet around WASP-160A, no transit-derived value for $\rho_{\star}$ was available. Our handful of RV measurements of WASP-160A are stable within $\sim 40 \mathrm{~m} \mathrm{~s}^{-1}$. We summarize the properties of WASP-160A and the WASP-160A+B binary in Table 9. Both stars appear to have similar masses and early $\mathrm{K}$ spectral types and their projected separation of $28.478948 \pm 2.5 \times 10^{-5}$ arcsec translates into a physical distance of $8060 \pm 101 \mathrm{au}$. As WASP-160B, also WASP-160A has a super-Solar metallicity, $([\mathrm{Fe} / \mathrm{H}]=0.19 \pm 0.09)$, consistent within uncertainties with the value found for WASP-160B. Even though we would expect the two object to be coeval, we find a slightly older age for WASP-160A from evolutionary models. While this reinforces the older age estimate for WASP-160A, the discrepancy found in our analysis between objects A and B is likely an artifact of the very limited available data on WASP-160A. 
Table 6. Basic properties and stellar parameters of the planet hosts based on spectroscopic analysis, evolutionary models and photometric variability. ${ }^{a}$ Derived from Gaia DR2 parallaxes (Gaia Collaboration et al. 2018) corrected for the $-80 \mu$ arcsec offset found by Stassun \& Torres (2018); ${ }^{b}$ Using Sestito \& Randich (2005); ${ }^{c}$ Internal model grid uncertainty; ${ }^{d}$ Estimated uncertainty accounting for different model grids; ${ }^{e}$ Using Barnes (2007).

\begin{tabular}{|c|c|c|c|c|}
\hline Parameter & WASP-147 & WASP-160B & WASP-164 & WASP-165 \\
\hline \multicolumn{5}{|l|}{ Basic parameters } \\
\hline RA (J2000) & 235645.97 & 055043.06 & 225929.62 & 235019.33 \\
\hline DEC (J2000) & -220911.39 & -273723.39 & -602651.97 & -170439.26 \\
\hline UCAC4 B [mag] & $12.96 \pm 0.04$ & $13.98 \pm 0.03$ & $13.32 \pm 0.03$ & $13.41 \pm 0.03$ \\
\hline UCAC4 V [mag] & $12.31( \pm<0.01)$ & $13.09 \pm 0.01$ & $12.62 \pm 0.01$ & $12.69 \pm 0.04$ \\
\hline 2MASS J [mag] & $11.174 \pm 0.023$ & $11.591 \pm 0.030$ & $11.365 \pm 0.024$ & $11.439 \pm 0.023$ \\
\hline 2MASS H [mag] & $10.907 \pm 0.025$ & $11.172 \pm 0.024$ & $11.040 \pm 0.024$ & $11.125 \pm 0.024$ \\
\hline 2MASS K [mag] & $10.857 \pm 0.025$ & $11.055 \pm 0.019$ & $10.959 \pm 0.021$ & $11.024 \pm 0.023$ \\
\hline GAIA DR2 ID & 2340919358581488768 & 2910755484609597312 & 6491038642006989056 & 2415410962124813056 \\
\hline GAIA G [mag] & 12.2 & 12.9 & 12.5 & 12.5 \\
\hline GAIA $G_{\mathrm{BP}}[\mathrm{mag}]$ & 12.5 & 13.3 & 12.8 & 12.9 \\
\hline GAIA $G_{\mathrm{RP}}[\mathrm{mag}]$ & 11.7 & 12.3 & 11.9 & 12.0 \\
\hline Distance $[\mathrm{pc}]^{a}$ & $426 \pm 14$ & $284 \pm 5$ & $322 \pm 7$ & $583 \pm 34$ \\
\hline \multicolumn{5}{|c|}{ Parameters from spectral analysis } \\
\hline Spectral type & G4 & K0V & $\mathrm{G} 2 \mathrm{~V}$ & G6 \\
\hline$T_{\text {eff }}[\mathrm{K}]$ & $5700 \pm 100$ & $5300 \pm 100$ & $5800 \pm 200$ & $5600 \pm 150$ \\
\hline$[\mathrm{Fe} / \mathrm{H}]$ & $+0.09 \pm 0.07$ & $+0.27 \pm 0.1$ & $0.0 \pm 0.2$ & $+0.33 \pm 0.13$ \\
\hline $\log g[\mathrm{cgs}]$ & $4.0 \pm 0.1$ & $4.6 \pm 0.1$ & $4.5 \pm 0.2$ & $3.9 \pm 0.2$ \\
\hline $\mathrm{V}_{\mathrm{rot}} \sin i_{*}\left[\mathrm{~km} \mathrm{~s}^{-1}\right]$ & $0.3_{-0.3}^{+0.8}$ & $\sim 0$ & $\sim 0$ & $\sim 0$ \\
\hline $\log A(\mathrm{Li})$ & $1.91 \pm 0.09$ & - & - & $2.21 \pm 0.14$ \\
\hline Lithium Age $^{b}[\mathrm{Gyr}]$ & $\geq 2$ & $\geq 0.5$ & - & $0.5-2$ \\
\hline \multicolumn{5}{|c|}{ Parameters from stellar evolutionary models } \\
\hline$M_{*}$ & $1.04( \pm 0.02)^{c} \pm 0.07^{d}$ & $0.87( \pm 0.02)^{c} \pm 0.07^{d}$ & $0.95( \pm 0.04)^{c} \pm 0.07^{d}$ & $1.25( \pm 0.04)^{c} \pm 0.07^{d}$ \\
\hline$R_{*}$ & $1.37( \pm 0.03)^{c} \pm 0.07^{d}$ & $0.87( \pm 0.01)^{c} \pm 0.07^{d}$ & $0.92( \pm 0.02)^{c} \pm 0.07^{d}$ & $1.65( \pm 0.06)^{c} \pm 0.07^{d}$ \\
\hline Age [Gyr] & $8.47 \pm 0.78$ & $9.75 \pm 2.28$ & $4.08 \pm 2.38$ & $4.77 \pm 0.92$ \\
\hline Gyrochronological age $^{e}[\mathrm{Gyr}]$ & - & - & $2.32_{-0.55}^{+0.98}$ & - \\
\hline
\end{tabular}

Table 7. Periodogram analysis of the WASP light curves for WASP-164. Observing dates are JD-2450000, $N$ is the number of observations used in the analysis, $a$ is the semi-amplitude of the best-fit sine wave at the period $P$ found in the periodogram with false-alarm probability FAP.

\begin{tabular}{rlrrrr}
\hline Camera & Dates & $N$ & $P[\mathrm{~d}]$ & $a[\mathrm{mmag}]$ & FAP \\
\hline \hline 221 & $5336-5515$ & 7916 & 17.790 & 0.006 & $<10^{-4}$ \\
221 & $5699-5881$ & 5623 & 17.730 & 0.007 & $<10^{-4}$ \\
221 & $6064-6106$ & 1004 & 1.014 & 0.004 & 0.93 \\
222 & $5352-5527$ & 7906 & 17.850 & 0.006 & $<10^{-4}$ \\
222 & $5716-5897$ & 5041 & 17.850 & 0.007 & $<10^{-4}$ \\
\hline
\end{tabular}

Table 8. Stellar mass and age estimates obtained with BAGEMASS. The mean and standard deviation of the posterior distributions are given together with the best-fit values in parentheses.

\begin{tabular}{lll}
\hline Star & Mass $\left[\boldsymbol{M}_{\odot}\right]$ & Age $[\mathrm{Gyr}]$ \\
\hline \hline WASP-147 & $1.08 \pm 0.07(1.05)$ & $8.4 \pm 1.9(9.1)$ \\
WASP-160B & $0.98 \pm 0.04(1.01)$ & $3.7 \pm 2.3(1.5)$ \\
WASP-164 & $0.96 \pm 0.07(1.02)$ & $4.7 \pm 3.3(2.0)$ \\
WASP-165 & $1.17 \pm 0.09(1.18)$ & $7.3 \pm 2.2(7.3)$ \\
\hline
\end{tabular}

\section{SYSTEM PARAMETERS}

\subsection{Modeling approach}

We carried out a global analysis of all follow-up photometry and RVs for each planetary system using the Markov Chain Monte Carlo (MCMC) framework described by Gillon, M. et al. (2012). In short, our model consists of a Keplerian for the RVs and the prescription of Mandel \& Agol (2002) for transit light curves. Next to the fitted ("jump") parameters listed in Table 10, the code allows for the inclusion of parametric baseline models in the form of polynomials (up to 4 th order) when fitting transit light curves. We tested a wide range of baseline models, including dependencies on time, airmass, stellar FWHM, coordinate shifts and sky background, and finally selected the appropriate solution for each light curve via Bayes factor comparison (e.g. Schwarz 1978). The best baseline models are listed in Table 5. For all objects, we tested for a non-zero eccentricity by running two sets of global analyses: one while fixing the eccentricity to zero and one fitting for it by including $\sqrt{e} \sin \omega$ and $\sqrt{e} \cos \omega$ as jump parameters. We found no significant evidence for a non-circular orbit for any of our targets.

To estimate excess noise, we calculated the $\beta_{r}$ and $\beta_{w}$ (Winn et al. 2008; Gillon et al. 2010) factors that compare the rms of the binned and unbinned residuals and multi- 

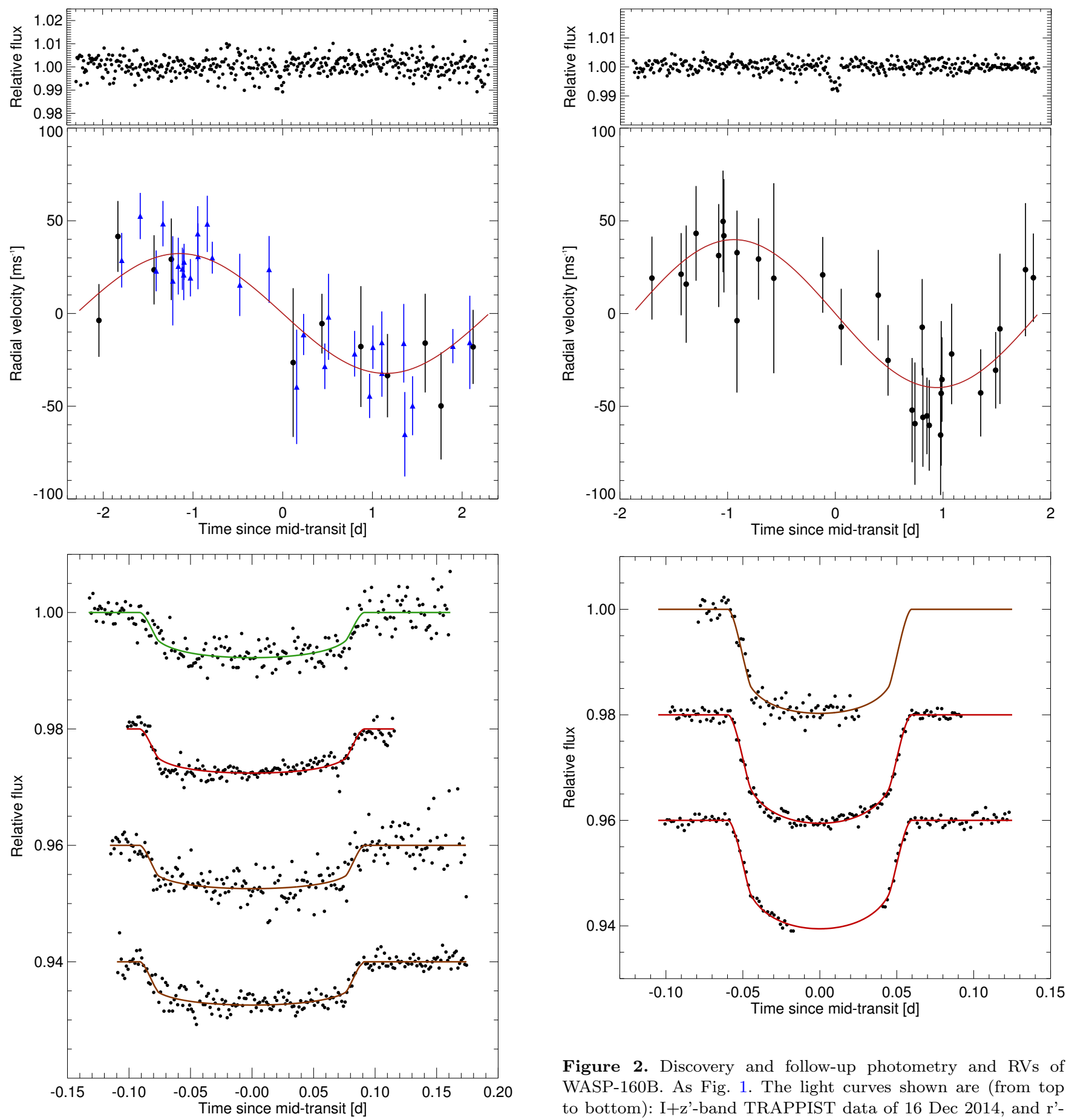

Figure 2. Discovery and follow-up photometry and RVs of WASP-160B. As Fig. 1. The light curves shown are (from top to bottom): I+z'-band TRAPPIST data of 16 Dec 2014, and r'band EulerCam data of 26 Dec 2015 and 02 Jan 2017. Note that the transit depth in the WASP light curve is reduced due to contamination from WASP-160A.

Figure 1. Discovery and follow-up photometry and RVs WASP-147. Top panel: WASP survey data, phase-folded on the period of WASP-147b and binned per 15 minutes. Middle panel: CORALIE RV data, where the pre-upgrade data are shown as blue triangles, and the post-upgrade data are shown as black filled circles. Bottom panel: Follow-up transit light curves, corrected for their respective baseline models and binned by two minutes. They are (from top to bottom): V-band TRAPPIST data of 05 Nov 2014, and r'-band EulerCam data of 11 Nov 2013, and blue-block TRAPPIST data of 19 Oct 2013 and 26 Sep 2013. The systematics seen in the 11 Nov 2013 light curve are due to cloud passages.

plied our error bars by their product before deriving the final parameter values. We find no excess "jitter" noise in the RVs and thus do not adapt the RV errors. We adopted a quadratic stellar limb-darkening law using coefficients interpolated from the tables by Claret \& Bloemen (2011). To use the most appropriate input stellar parameters, we use the information extracted from the stellar spectra, paired with the Gaia DR2 (Gaia Collaboration et al. 2018) data as described in Section 3. After carrying out an initial analysis to 

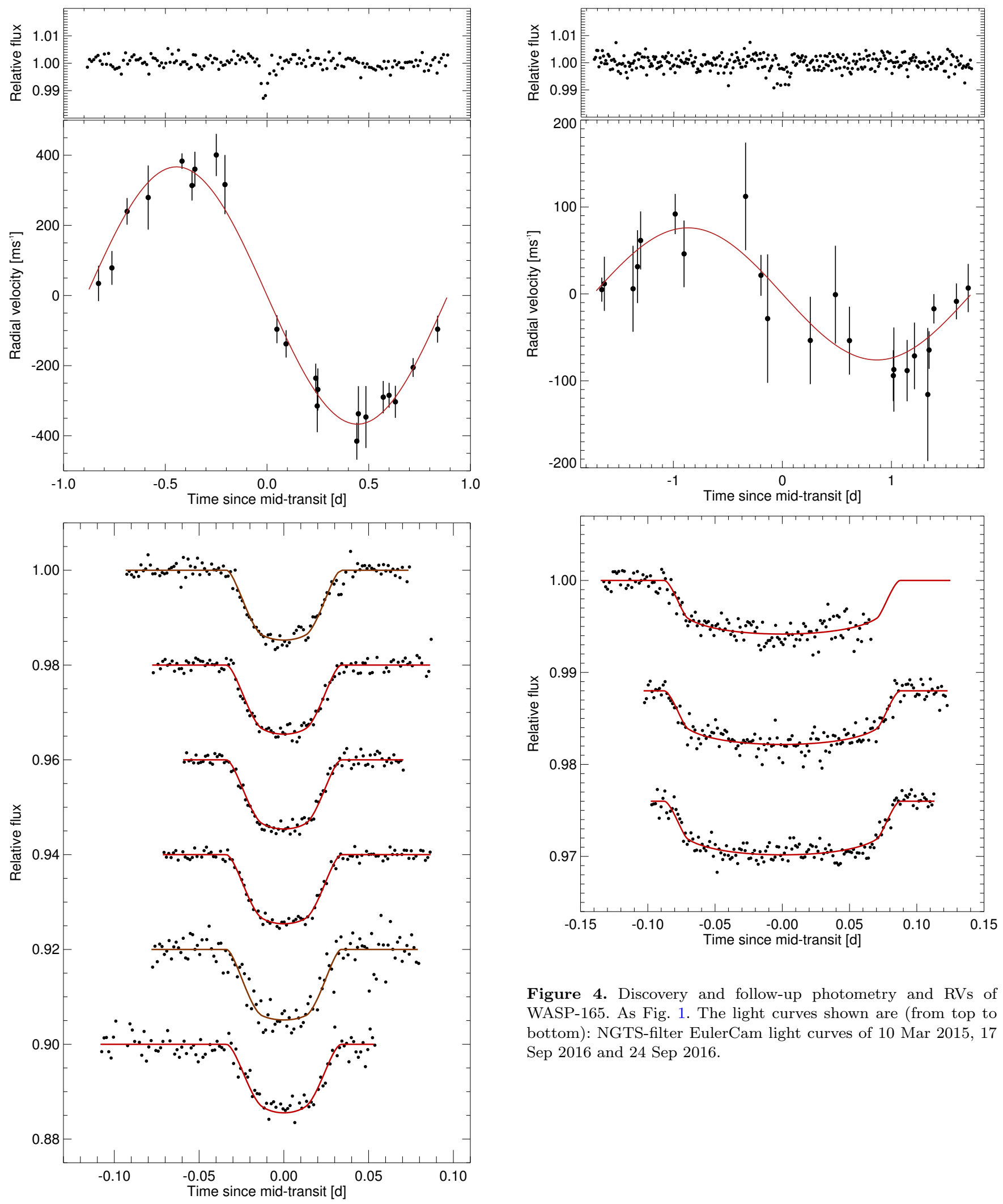

Figure 4. Discovery and follow-up photometry and RVs of WASP-165. As Fig. 1. The light curves shown are (from top to bottom): NGTS-filter EulerCam light curves of 10 Mar 2015, 17 Sep 2016 and 24 Sep 2016.

Figure 3. Discovery and follow-up photometry and RVs of WASP-164. As Fig. 1. The light curves shown are (from top to bottom): blue-block TRAPPIST data of 29 Jun 2015, r'-band EulerCam data of 31 Jul 2015, 16 Aug 2015 and 25 Aug 2015, I+z'band TRAPPIST data of 10 Sep 2016 and and R-band SAAO data of 16 Oct 2016.

measure transit-based stellar mean densities needed to constrain the evolutionary models, we placed normal priors on $M_{*},[\mathrm{Fe} / \mathrm{H}]_{*}$ and $T_{\text {eff }}$ centered on the values inferred in Section 3 , with a width of the quoted $1-\sigma$ uncertainties. The four objects under study are revealed to be gas giants, two of them being classical hot Jupiters, while two have masses near that of Saturn. 

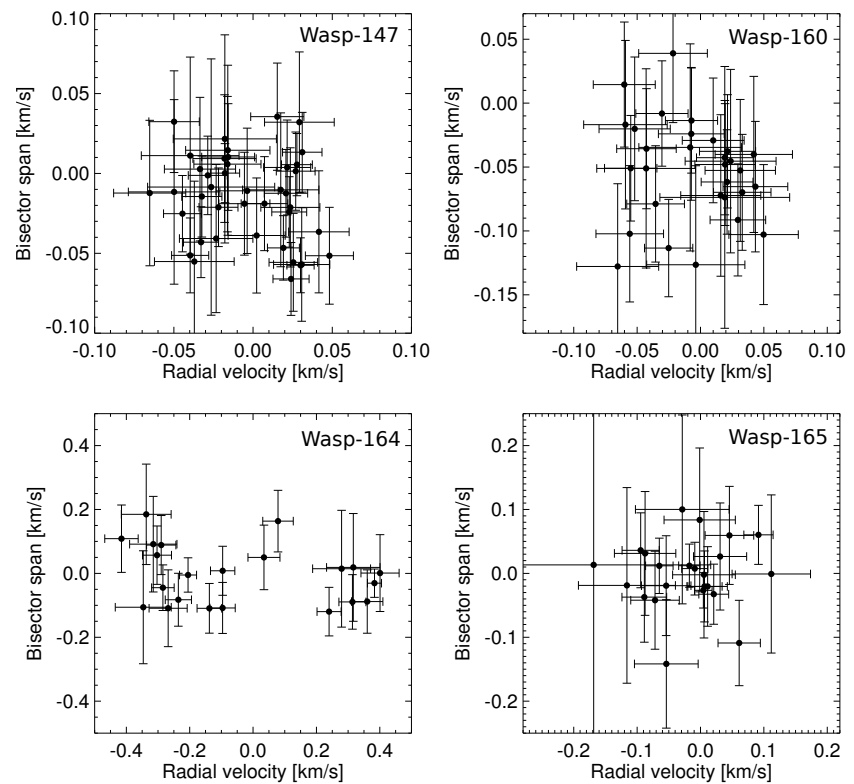

Figure 5. Bisector spans against RV of our targets. The RVs have been corrected for the systemic velocities given in Table 10 .

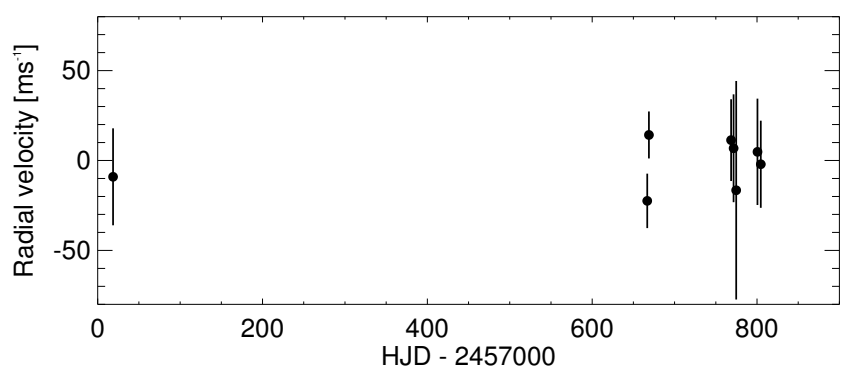

Figure 6. CORALIE RVs of WASP-160A.

\subsection{WASP-147}

WASP-147b is a Saturn-mass $\left(M=0.27 M_{J}\right)$ planet orbiting a G4 star with a period of 4.6 days. The system appears to be old, with the $1.04 M_{\odot}$ host having started to evolve off the main sequence. Stellar evolutionary modeling places the star's age at $8.5 \pm 0.8 \mathrm{Gyr}$, and its old age is corroborated by a low Lithium abundance that is in accordance with measurements for stars aged 2 Gyrs or more (Sestito \& Randich 2005) and the absence of activity indicators such as excess RV stellar noise and rotational variability. The planet is one of the more strongly irradiated planets of its mass range. Considering the mass-incident flux plane shown in Fig. 9, WASP-147b is located near the inner tip of the triangular sub-Saturn desert (Mazeh et al. 2005; Szabó \& Kiss 2011; Mazeh et al. 2016), which appears to be created by erosion of planetary atmospheres due to stellar irradiation (Lammer et al. 2003; Baraffe et al. 2006). Being a low-mass, lowdensity planet, WASP-147b is a good target for transmission spectroscopy. One atmospheric scale height translates to a predicted change in the transit depth of $249 \mathrm{ppm}$, well within the precision of ground- and space-based transmission spec-
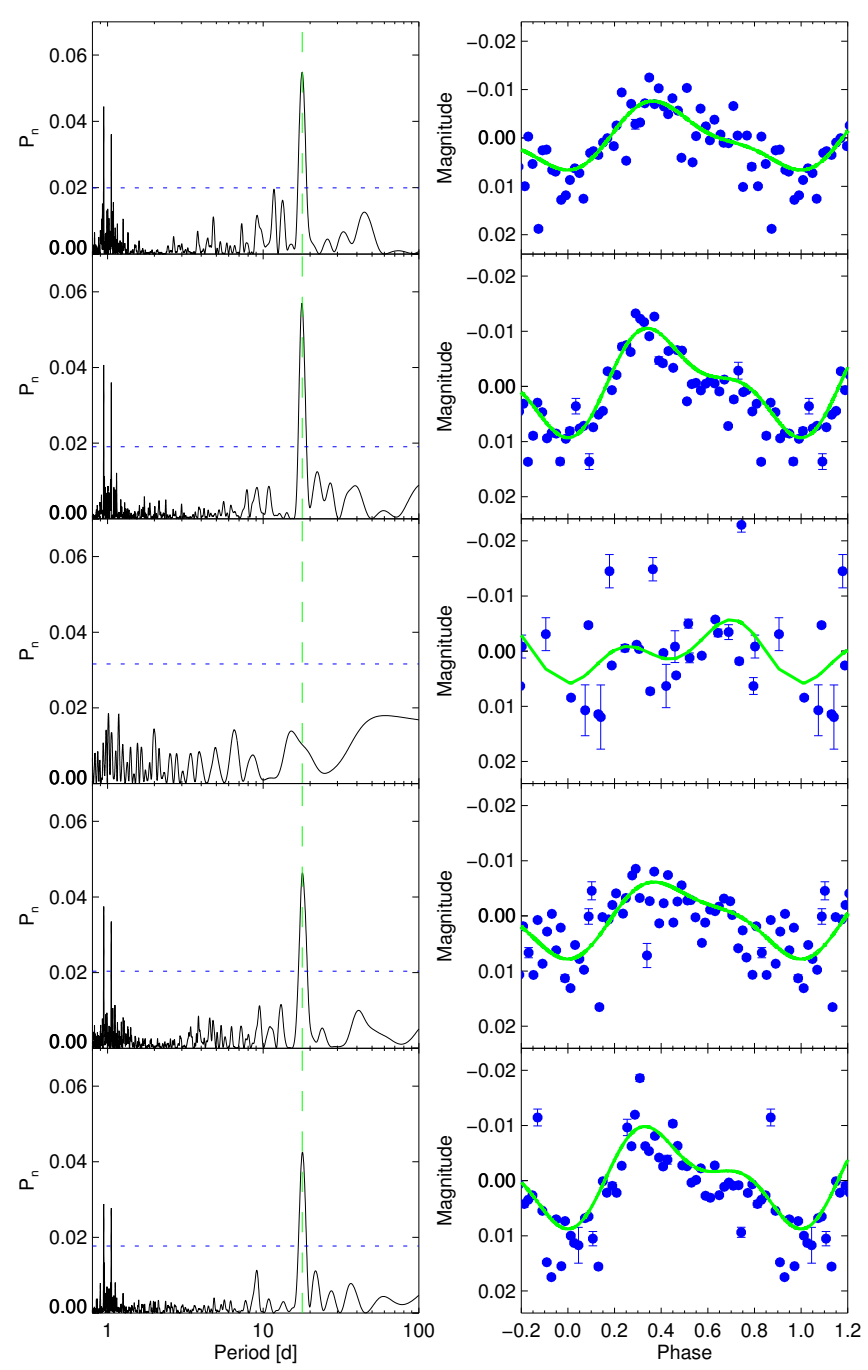

Figure 7. Left: Periodograms of the WASP light curves for WASP-164. Horizontal lines indicate false-alarm probability levels 0.1, 0.01 and 0.001. Right: Light curves phase-binned on the assumed rotation period of 17.81 days (points) with second-order harmonic series fit by least squares (lines). Data are plotted by season and camera in the same order top-to-bottom as in Table 7.

tra (e.g. Kreidberg et al. 2015; Sing et al. 2016; Lendl et al. 2017; Sedaghati et al. 2017).

\subsection{WASP-160B}

Similar to WASP-147b, WASP-160Bb is also a near Saturnmass $\left(M_{p}=0.28 M_{J}\right)$ object, however this planet orbits a cooler K0V star in a wide $(28.5$ arcsec) near equal-mass binary with a period of 3.8 days. The stellar age is estimated to be approximately 10 Gyrs from evolutionary models, and a non-detection of Lithium supports the object's old age. In contrast to WASP-147, the later-type WASP-160B still resides firmly on the main sequence. Both planets share near-identical mass and radius, however WASP-160Bb orbits a very metal-rich star $([\mathrm{Fe} / \mathrm{H}]=0.27 \pm 0.1)$, while WASP147 's metallicity is near-Solar. WASP- $160 \mathrm{Bb}$ receives less 

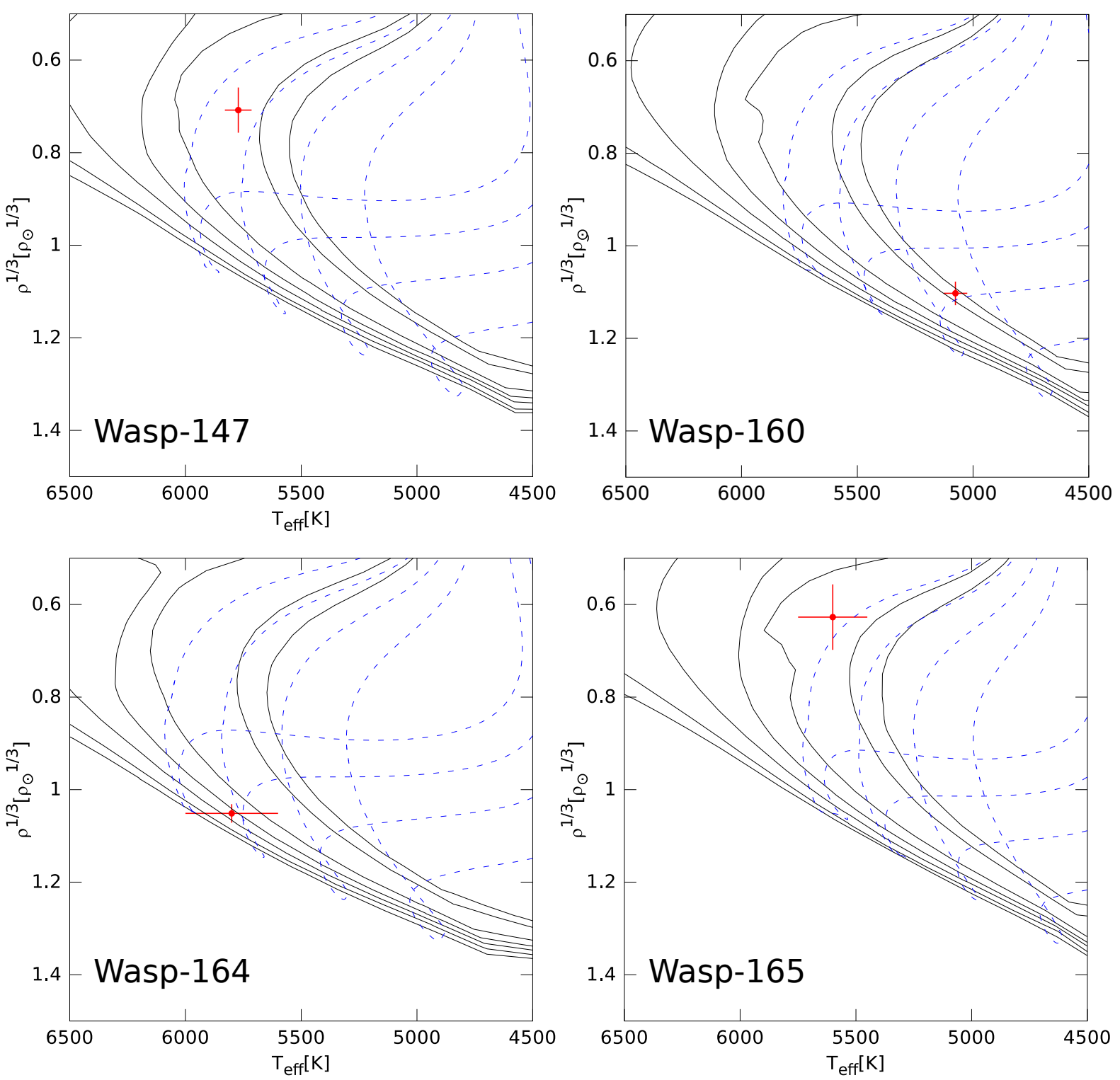

Figure 8. Placement of the planet host stars in the HR diagram. In each panel, theoretical models corresponding to the metallicity of that star are shown. Solid black lines are representative of isochrones: from left to right $0.5,1,2,3.2,5,10$ and 12.6 Gyr models are represented. Dashed blue lines are representative of evolutionary tracks: from left to right $1.1,1,0.9$ and $0.8 M_{\odot}$ models are represented.

stellar irradiation than the bulk of hot Jupiters (see Fig. 9), which translates to a moderate equilibrium temperature of approx. $1100 \mathrm{~K}$. Prospects for studying this object's atmospheric transmission spectrum are excellent, as one atmospheric scale height translates to a radius variation of $338 \mathrm{ppm}$. To date, the only hot Jupiter orbiting a more metal-rich host with a characterized transmission spectrum is XO-2 (Burke et al. 2007; Sing et al. 2011), for which both $\mathrm{Na}$ and $\mathrm{K}$ have been detected.

\subsection{WASP-164}

WASP-164b is a massive $\left(M_{p}=2.13 M_{J}\right)$ planet on a closein orbit around a G2V star. Owing to the limited $\mathrm{S} / \mathrm{N}$ of the follow-up spectra obtained, the stellar properties are somewhat more uncertain than for the other objects pre- sented here. However, rotational modulation with a period of $17.81 \pm 0.03$ days seen in the survey light curve indicates a relatively young age of $2.3_{-0.5}^{+1}$ Gyr and evolutionary models confirm the star being located on the main sequence. The planet is rather massive compared to the bulk of hot Jupiters and orbits its host star with a short orbital period of 1.78 days at a separation of 2.74 times its Roche limit. This is consistent with expectations from high-eccentricity migration mechanisms (Ford \& Rasio 2006). Being rather massive and close-in, the planet may be further undergoing orbital decay through tidal dissipation (e.g. Rasio et al. 1996; Levrard et al. 2009; Matsumura et al. 2010). Using a value of $\log Q_{s}^{\prime}=8.26 \pm 0.14$ (Collier Cameron \& Jardine 2018), we find a remaining lifetime of $13.0 \pm 4.6 \mathrm{Gyr}$. The planetary orbit is thus not expected to shrink drastically due to tidal dissipation over the star's main-sequence lifetime. The planet's close-in orbital period is however advantageous for 
Table 9. Properties of WASP-160A and the WASP-160A+B binary ${ }^{a}$ assuming a macroturbulence of $2.43 \mathrm{~km} \mathrm{~s}^{-1}$ from Doyle et al. (2014). ${ }^{b}$ from stellar evolutionary models, ${ }^{c}$ From Gaia DR2 and CORALIE, ${ }^{d}$ computed from parallaxes corrected for the $-80 \mu$ arcsec offset found by Stassun \& Torres (2018).

\begin{tabular}{|c|c|c|}
\hline \multicolumn{3}{|c|}{ WASP-160A stellar properties } \\
\hline RA (J2000) & \multicolumn{2}{|c|}{055044.7} \\
\hline DEC (J2000) & \multicolumn{2}{|c|}{-273705.0} \\
\hline UCAC4 B [mag] & \multicolumn{2}{|c|}{$13.452 \pm 0.04$} \\
\hline UCAC4 V [mag] & \multicolumn{2}{|c|}{$12.677 \pm 0.02$} \\
\hline 2MASS J [mag] & \multicolumn{2}{|c|}{$11.300 \pm 0.026$} \\
\hline 2MASS H [mag] & \multicolumn{2}{|c|}{$10.937 \pm 0.024$} \\
\hline 2MASS K [mag] & \multicolumn{2}{|c|}{$10.831 \pm 0.019$} \\
\hline GAIA DR2 ID & \multicolumn{2}{|c|}{2910755484609594368} \\
\hline GAIA G [mag] & \multicolumn{2}{|c|}{12.5} \\
\hline GAIA $G_{\mathrm{BP}}[\mathrm{mag}]$ & \multicolumn{2}{|c|}{12.9} \\
\hline GAIA $G_{\mathrm{RP}}[m a g]$ & \multicolumn{2}{|c|}{11.9} \\
\hline Spectral Type & \multicolumn{2}{|c|}{ K0V } \\
\hline$T_{\text {eff }}[\mathrm{K}]$ & \multicolumn{2}{|c|}{$5300 \pm 150$} \\
\hline $\log g[\mathrm{cgs}]$ & \multicolumn{2}{|c|}{$4.5 \pm 0.2$} \\
\hline$[\mathrm{Fe} / \mathrm{H}]$ & \multicolumn{2}{|c|}{$0.19 \pm 0.09$} \\
\hline $\mathrm{V}_{\mathrm{rot}} \sin i_{*}\left[\mathrm{~km} \mathrm{~s}^{-1}\right]^{a}$ & \multicolumn{2}{|c|}{$0.4 \pm 0.2$} \\
\hline $\log A(\mathrm{Li})$ & \multicolumn{2}{|c|}{-} \\
\hline $\operatorname{Mass}\left[\boldsymbol{M}_{\odot}\right]$ & \multicolumn{2}{|c|}{$0.89 \pm 0.07$} \\
\hline Radius $\left[R_{\odot}\right.$ & \multicolumn{2}{|c|}{$0.95 \pm 0.07$} \\
\hline Age $[\mathrm{Gyr}]^{b}$ & \multicolumn{2}{|c|}{$11.2 \pm 1.6$} \\
\hline \multicolumn{3}{|l|}{ Binary properties ${ }^{c}$} \\
\hline & WASP-160A & WASP-160B \\
\hline Proper motion RA [mas] & $26.85 \pm 0.03$ & $26.97 \pm 0.03$ \\
\hline Proper motion DEC [mas] & $-34.82 \pm 0.04$ & $-34.83 \pm 0.04$ \\
\hline Parallax [mas] & $3.46 \pm 0.03$ & $3.44 \pm 0.02$ \\
\hline Distance $[\mathrm{pc}]^{d}$ & $282 \pm 5$ & $284 \pm 5$ \\
\hline \multirow[t]{2}{*}{ System RV $\left[\mathrm{km} \mathrm{s}^{-1}\right]$} & -6.1587 & -6.1421 \\
\hline & 0.0075 & 0.0012 \\
\hline \multicolumn{3}{|c|}{ Position angle of B with respect to A [deg] $\quad-129.96$} \\
\hline Separation $[\operatorname{arcsec}]$ & \multicolumn{2}{|c|}{$28.478948 \pm 2.5 \times 10^{-5}$} \\
\hline Separation $[\mathrm{au}]$ & \multicolumn{2}{|c|}{$8060 \pm 101$} \\
\hline
\end{tabular}

the object's atmospheric characterization. Indeed, WASP164b makes for a promising target for characterization via emission spectroscopy, with a predicted occultation depth in $\mathrm{H}$ band between $450 \mathrm{ppm}$ (full redistribution) and $1125 \mathrm{ppm}$ (immediate reradiation).

\subsection{WASP-165}

WASP-165b is a classical hot Jupiter. With an orbital period of 3.4 days, it lies near the peak of the hot Jupiter period distribution while its mass $\left(M_{P}=0.66 M_{J}\right)$ and radius $\left(R_{P}=1.26 R_{J}\right)$ are typical for this class of planet (see Fig. 9). With respect to planetary mass, radius and period, WASP$165 \mathrm{~b}$ is near-identical to the extremely well-studied object HD 209458b (Mazeh et al. 2000; Charbonneau et al. 2000; Henry et al. 2000), however WASP-165b is more strongly irradiated as its $M_{*}=1.25 M_{\odot}$ G6 host has started evolving off the main sequence. The system's age estimate from evolutionary models is $4.8 \pm 1 \mathrm{Gyr}$, albeit the object's Lithium abundance suggests a slightly younger age of $0.5-2$ Gyr. This discrepancy is most likely due to the limited $\mathrm{S} / \mathrm{N}$ of
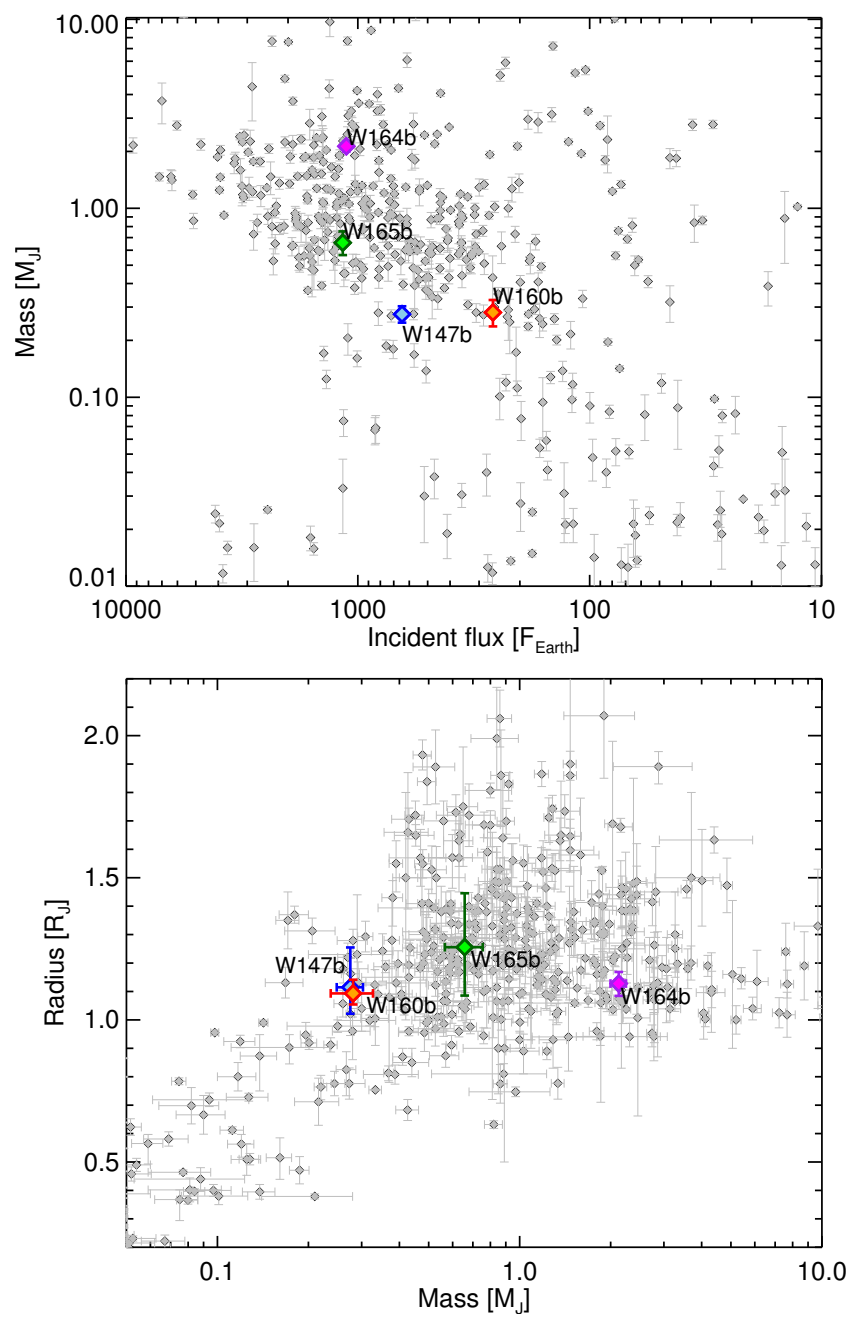

Figure 9. Top: Planetary masses against incident flux for known exoplanets. Only planets with well-measured masses and radii (relative uncertainties smaller than 50\%) are shown. Our newlydiscovered objects are shown in color and labelled. Bottom: Planetary mass-radius diagram. Sample selection and designation of our targets as above.

our spectra. We consider the age estimate from evolutionary models as more accurate, as these contain the stellar mean density inferred from the high-precision follow-up light curves. As WASP-160B, also WASP-165 has a remarkably high metallicity of $[\mathrm{Fe} / \mathrm{H}]=0.33 \pm 0.13$.

\section{SUMMARY}

We present the discovery of four transiting hot Jupiters by WASP-South. WASP-147b and WASP-160Bb are Saturnmass planets with near-identical radii but contrasting stellar metallicities and planetary equilibrium temperatures: the 1400 K WASP-147b orbits a Solar-metallicity G4 star, while the $1100 \mathrm{~K}$ WASP-160Bb orbits a metal-rich K0 star in a near equal-mass binary. Both objects are promising targets for atmospheric characterization via transmission spectroscopy. WASP-164b is a massive $\left(2.1 M_{J}\right)$ planet on a short $(1.8 \mathrm{~d})$ orbital period, and a potential target for emission 
Table 10. Planetary and stellar parameters from a global MCMC analysis.

${ }^{a}$ Equilibrium temperature, assuming $A_{B}=0$ and $F=1$ (Seager et al. 2005).

\begin{tabular}{|c|c|c|c|c|}
\hline Parameter & WASP-147 & WASP-160B & WASP-164 & WASP-165 \\
\hline \multicolumn{5}{|l|}{ Jump parameters } \\
\hline Transit depth, $\Delta F$ & $0.00640_{-0.00059}^{+0.00062}$ & $0.01663_{-0}^{+0.000038}$ & $0.01542 \pm 0.00033$ & $0.00544_{-0.00059}^{+0.00067}$ \\
\hline$b^{\prime}=a * \cos \left(i_{p}\right)\left[R_{*}\right]$ & $0.31_{-0.21}^{+0.19}$ & $0.20_{-0.12}^{+0.10}$ & $0.8216_{-0.0091}^{+0.0084}$ & $0.53_{-0.22}^{+0.11}$ \\
\hline Transit duration, $T_{14}[\mathrm{~d}]$ & $0.1831_{-0.0033}^{+0.0041}$ & $0.11851_{-0.00078}^{+0.00094}$ & $0.06682_{-0.00084}^{+0.000085}$ & $0.1740_{-0.0034}^{+0.0040}$ \\
\hline Mid-transit time, [BJD] - 2450000 & $6562.5950 \pm 0.0013$ & $7383.65494 \pm 0.00021$ & $7203.85378 \pm 0.00020$ & $7649.71142 \pm 0.00093$ \\
\hline Period, $P[\mathrm{~d}]$ & $4.60273 \pm 0.000027$ & $3.7684952 \pm 0.0000035$ & $1.7771255 \pm 0.0000028$ & $3.465509 \pm 0.000023$ \\
\hline$K_{2}=K \sqrt{1-e^{2}} P^{1 / 3}\left[\mathrm{~ms}^{-1} \mathrm{~d}^{1 / 3}\right]$ & $54.4_{-4.6}^{+4.7}$ & $62.1_{-9.5}^{+9.2}$ & $445 \pm 15$ & $115 \pm 16$ \\
\hline Stellar mass, $M_{*}\left[M_{\odot}\right]$ & $1.044_{-0.073}^{+0.070}$ & $0.87_{-0.068}^{+0.071}$ & $0.946_{-0.071}^{+0.067}$ & $1.248_{-0.070}^{+0.072}$ \\
\hline Stellar eff. temperature, $T_{\text {eff }}[\mathrm{K}]$ & $5702 \pm 100$ & $5298 \pm 99$ & $5806_{-200}^{+190}$ & $5599 \pm 150$ \\
\hline Stellar metallicity, $[\mathrm{Fe} / \mathrm{H}]_{*}$ & $0.092_{-0.071}^{+0.069}$ & $0.27 \pm 0.10$ & $-0.01_{-0.20}^{+0.19}$ & $0.33 \pm 0.13$ \\
\hline$c_{1, \mathrm{~V}}=2 u_{1, \mathrm{~V}}+u_{2, \mathrm{~V}}$ & $1.198_{-0.050}^{+0.051}$ & & & \\
\hline$c_{2, \mathrm{~V}}=u_{1, \mathrm{~V}}-2 u_{2, \mathrm{~V}}$ & $-0.014 \pm 0.040$ & & & \\
\hline$c_{1, \mathrm{R}}=2 u_{1, \mathrm{R}}+u_{2, \mathrm{R}}$ & & & $1.042_{-0.082}^{+0.081}$ & \\
\hline$c_{2, \mathrm{R}}=u_{1, \mathrm{R}}-2 u_{2, \mathrm{R}}$ & & & $-0.178_{-0.056}^{+0.053}$ & \\
\hline$c_{1, \mathrm{r}^{\prime}}=2 u_{1, \mathrm{r}^{\prime}}+u_{2, \mathrm{r}^{\prime}}$ & $1.079_{-0.044}^{+0.046}$ & $1.200 \pm 0.042$ & $1.022_{-0.074}^{-0.0076}$ & \\
\hline$c_{2, \mathrm{r}^{\prime}}=u_{1, \mathrm{r}^{\prime}}-2 u_{2, \mathrm{r}^{\prime}}$ & $-0.133_{-0.032}^{+0.034}$ & $0.093 \pm 0.036$ & $-0.178_{-0.059}^{+0.058}$ & \\
\hline$c_{1, \mathrm{NGTS}}=2 u_{1, \mathrm{NGTS}}+u_{2, \mathrm{NGTS}}$ & & & & $1.09 \pm 0.10$ \\
\hline$c_{2, \mathrm{NGTS}}=u_{1, \mathrm{NGTS}}-2 u_{2, \mathrm{NGTS}}$ & & & & $0.02 \pm 0.11$ \\
\hline$c_{1, \mathrm{I}+\mathrm{z}^{\prime}}=2 u_{1, \mathrm{I}+\mathrm{z}^{\prime}}+u_{2, \mathrm{I}+\mathrm{z}^{\prime}}$ & & $0.94 \pm 0.10$ & $0.88 \pm 0.10$ & \\
\hline$c_{2, \mathrm{I}+\mathrm{z}^{\prime}}=u_{1, \mathrm{I}+\mathrm{z}^{\prime}}-2 u_{2, \mathrm{I}+\mathrm{z}^{\prime}}$ & & $-0.07 \pm 0.11$ & $-0.14_{-0.11}^{+0.10}$ & \\
\hline$c_{1, \mathrm{BB}}=2 u_{1, \mathrm{BB}}+u_{2, \mathrm{BB}}$ & $0.99 \pm 0.10$ & & $0.99 \pm 0.10$ & \\
\hline$c_{2, \mathrm{BB}}=u_{1, \mathrm{BB}}-2 u_{2, \mathrm{BB}}$ & $0.00 \pm 0.11$ & & $-0.07 \pm 0.11$ & \\
\hline \multicolumn{5}{|l|}{ Deduced parameters } \\
\hline RV amplitude, $K\left[\mathrm{~m} \mathrm{~s}^{-1}\right]$ & $32.7 \pm 2.8$ & $39.9_{-6.1}^{+5.9}$ & $367 \pm 12$ & $76 \pm 10$ \\
\hline $\begin{array}{l}\text { RV zero point (pre-upgrade), } \gamma_{\text {COR1 }} \\
{\left[\mathrm{km} \mathrm{s}^{-1}\right]}\end{array}$ & $-1.63849 \pm 0.00061$ & & & \\
\hline RV zero point, $\gamma_{\mathrm{COR} 2}\left[\mathrm{~km} \mathrm{~s}^{-1}\right]$ & $-1.617011_{-0.000066}^{+0.000069}$ & $-6.1421 \pm 0.0012$ & $12.262676_{0.000091}^{+0.00094}$ & $25.6557 \pm 0.0012$ \\
\hline Planetary radius, $R_{p}\left[R_{J}\right]$ & $1.115_{-0.093}^{+0.0 .000066}$ & $1.090_{-0.041}^{+0.047}$ & $1.128_{-0.043}^{+0.041}$ & $1.26_{-0.17}^{+0.19}$ \\
\hline Planetary mass, $M_{p}\left[M_{J}\right]$ & $0.275_{-0.027}^{+0.028}$ & $0.278_{-0.045}^{+0.041}$ & $2.13_{-0.13}^{-0.12}$ & $0.658_{-0.092}^{+0.1997}$ \\
\hline Planetary mean density, $\rho_{p}\left[\rho_{J}\right]$ & $0.198_{-0.060}^{+0.027}$ & $0.214_{-0.038}^{-0.045}+0.039$ & $1.48_{-0.13}^{+0.13}$ & $\begin{array}{l}0.33_{-0.11}^{+0.092} \\
0.19\end{array}$ \\
\hline Planetary grav. acceleration, $\log g_{p}[\mathrm{cgs}]$ & $2.74_{-0.11}^{+0.060}$ & $2.763_{-0.077}^{+0.038}$ & $3.619_{-0.028}^{+0.029}$ & $3.01_{-0.13}^{+0.11}$ \\
\hline Planetary eq. temperature, $T_{e q}[\mathrm{~K}]^{a}$ & $1404_{-43}^{+69}$ & $1119_{-23}^{+25}$ & $1610_{-53}^{+58}$ & $1624_{-89}^{+93}$ \\
\hline Orbital semi-major axis, $a$ [au] & $0.0549_{-0.0013}^{+0.0012}$ & $0.0452 \pm 0.0012$ & $0.02818_{-0.50072}^{+0.00065}$ & $0.04823_{-0.00092}^{+0.00091}$ \\
\hline$a / R_{*}$ & $8.29_{-0.74}^{+0.4013}$ & $11.25_{-0.31}^{+0.19}$ & $6.50 \pm 0.13$ & $5.93_{-0.55}^{+0.67092}$ \\
\hline$R_{p} / R_{*}$ & $0.0800 \pm 0.0038$ & $0.1290_{-0.0015}^{+0.0017}$ & $0.1242 \pm 0.0013$ & $0.0738_{-0.0041}^{+0.0044}$ \\
\hline Inclination, $i_{p}[\mathrm{deg}]$ & $87.9_{-1.6}^{+1.5}$ & $88.97_{-0.57}^{+0.63}$ & $82.73_{-0.21}^{+0.22}$ & $84.9_{-1.7}^{+2.541}$ \\
\hline Eccentricity, $e$ & $0(<0.19$ at $1 \sigma)$ & $0(<0.22$ at $1 \sigma)$ & $0(<0.09$ at $1 \sigma)$ & $0(<0.14$ at $1 \sigma)$ \\
\hline Stellar radius, $R_{*}\left[R_{\odot}\right]$ & $1.429_{-0.076}^{+0.14}$ & $0.868_{-0.028}^{+0.031}$ & $0.932_{-0.030}^{+0.028}$ & $1.75 \pm 0.18$ \\
\hline Stellar mean density, $\rho_{*}\left[\rho_{\odot}\right]$ & $0.361_{-0.088}^{+0.076}$ & $1.34_{-0.11}^{+0.028}$ & $1.165_{-0.067}^{+0.030}$ & $0.233_{-0.059}^{+0.089}$ \\
\hline Limb-darkening coefficient, $u_{1, \mathrm{~V}}$ & $0.477 \pm 0.027$ & & & \\
\hline Limb-darkening coefficient, $u_{2, \mathrm{~V}}$ & $0.245 \pm 0.018$ & & & \\
\hline Limb-darkening coefficient, $u_{1, \mathrm{R}}$ & & & $0.381 \pm 0.040$ & \\
\hline Limb-darkening coefficient, $u_{2, \mathrm{R}}$ & & & $0.280_{-0.020}^{+0.021}$ & \\
\hline Limb-darkening coefficient, $u_{1, \mathrm{r}^{\prime}}$ & $0.405_{-0.023}^{+0.024}$ & $0.499 \pm 0.022$ & $0.373 \pm 0.038$ & \\
\hline Limb-darkening coefficient, $u_{2, \mathrm{r}^{\prime}}$ & $0.269 \pm 0.015$ & $0.203 \pm 0.017$ & $0.276_{-0.023}^{+0.022}$ & \\
\hline Limb-darkening coefficient, $u_{1, \mathrm{NGTS}}$ & & & & $0.440 \pm 0.056$ \\
\hline Limb-darkening coefficient, $u_{2, \text { NGTS }}$ & & & & $0.210 \pm 0.058$ \\
\hline Limb-darkening coefficient, $u_{1, \mathrm{I}+\mathrm{z}^{\prime}}$ & & $0.363 \pm 0.053$ & $0.323_{-0.047}^{+0.048}$ & \\
\hline Limb-darkening coefficient, $u_{2, \mathrm{I}+\mathrm{z}^{\prime}}$ & & $0.215 \pm 0.056$ & $\begin{array}{l}-0.047 \\
0.232_{-0.047}^{+0.049}\end{array}$ & \\
\hline Limb-darkening coefficient, $u_{1, \mathrm{BB}}$ & $0.398 \pm 0.053$ & & $0.384_{-0.048}^{+0.047}$ & \\
\hline Limb-darkening coefficient, $u_{2, \mathrm{BB}}$ & $0.196 \pm 0.055$ & & $0.224_{-0.048}^{+0.050}$ & \\
\hline
\end{tabular}


spectroscopy. Finally, WASP-165b is a classical hot Jupiter orbiting a metal-rich host.

\section{ACKNOWLEDGEMENTS}

WASP-South is hosted by the South African Astronomical Observatory and we are grateful for their ongoing support and assistance. Funding for WASP comes from consortium universities and from the UK's Science and Technology Facilities Council. TRAPPIST is funded by the Belgian Fund for Scientific Research (Fond National de la Recherche Scientifique, FNRS) under the grant FRFC 2.5.594.09.F, with the participation of the Swiss National Science Fundation (SNF). MG is a F.R.S.-FNRS Senior Research Associate. The research leading to these results has received funding from the European Research Council under the FP/2007-2013 ERC Grant Agreement 336480, from the ARC grant for Concerted Research Actions financed by the Wallonia-Brussels Federation, and from the Balzan Foundation. L.D. acknowledges support from the Gruber Foundation Fellowship. M.L. acknowledges support from the Austrian Research Promotion Agency (FFG) under project 859724 "GRAPPA".

\section{REFERENCES}

Bakos G., Noyes R. W., Kovács G., Stanek K. Z., Sasselov D. D., Domsa I., 2004, PASP, 116, 266

Bakos G. Á., et al., 2010, ApJ, 710, 1724

Baraffe I., Alibert Y., Chabrier G., Benz W., 2006, A\&A, 450, 1221

Baranne A., et al., 1996, A\&AS, 119, 373

Barnes S. A., 2007, ApJ, 669, 1167

Barnes S. A., 2010, ApJ, 722, 222

Bonfanti A., Ortolani S., Piotto G., Nascimbeni V., 2015, A\&A, 575, A18

Bonfanti A., Ortolani S., Nascimbeni V., 2016, A\&A, 585, A5

Bressan A., Marigo P., Girardi L., Salasnich B., Dal Cero C., Rubele S., Nanni A., 2012, MNRAS, 427, 127

Burke C. J., et al., 2007, ApJ, 671, 2115

Charbonneau D., Brown T. M., Latham D. W., Mayor M., 2000, ApJ, 529, L45

Charbonneau D., Brown T. M., Noyes R. W., Gilliland R. L., 2002, ApJ, 568, 377

Charbonneau D., et al., 2005, ApJ, 626, 523

Chen Y., Girardi L., Bressan A., Marigo P., Barbieri M., Kong X., 2014, MNRAS, 444, 2525

Claret A., Bloemen S., 2011, A\&A, 529, A75

Collier Cameron A., Jardine M., 2018, preprint, (arXiv: 1801.10561)

Collier Cameron A., et al., 2006, MNRAS, 373, 799

Collier Cameron A., et al., 2007, MNRAS, 380, 1230

Doyle A. P., et al., 2013, MNRAS, 428, 3164

Doyle A. P., Davies G. R., Smalley B., Chaplin W. J., Elsworth Y., 2014, MNRAS, 444, 3592

Ford E. B., Rasio F. A., 2006, ApJ, 638, L45

Gaia Collaboration Brown A. G. A., Vallenari A., Prusti T., de Bruijne J. H. J., Babusiaux C., Bailer-Jones C. A. L., 2018, preprint, (arXiv: 1804.09365)

Gillon, M. et al., 2012, A\&A, 542, A4

Gillon M., et al., 2010, A\&A, 511, A3

Gillon M., Jehin E., Magain P., Chantry V., Hutsemékers D., Manfroid J., Queloz D., Udry S., 2011, Detection and Dynamics of Transiting Exoplanets, St. Michel l'Observatoire,
France, Edited by F. Bouchy; R. Díaz; C. Moutou; EPJ Web of Conferences, Volume 11, id.06002, 11, 6002

Hebb L., et al., 2009, ApJ, 693, 1920

Hellier C., et al., 2011, A\&A, 535, L7

Henry G. W., Marcy G. W., Butler R. P., Vogt S. S., 2000, ApJ, 529, L41

Jehin E., et al., 2011, The Messenger, 145, 2

Kreidberg L., et al., 2015, ApJ, 814, 66

Lammer H., Selsis F., Ribas I., Guinan E. F., Bauer S. J., Weiss W. W., 2003, ApJ, 598, L121

Lendl M., et al., 2012, A\&A, 544, A72

Lendl M., Cubillos P. E., Hagelberg J., Müller A., Juvan I., Fossati L., 2017, preprint, (arXiv:1708.05737)

Levrard B., Winisdoerffer C., Chabrier G., 2009, ApJ, 692, L9

Mandel K., Agol E., 2002, ApJ, 580, L171

Matsumura S., Peale S. J., Rasio F. A., 2010, ApJ, 725, 1995

Maxted P. F. L., et al., 2011, PASP, 123, 547

Maxted P. F. L., Serenelli A. M., Southworth J., 2015, A\&A, 575, A36

Mazeh T., et al., 2000, ApJ, 532, L55

Mazeh T., Zucker S., Pont F., 2005, MNRAS, 356, 955

Mazeh T., Holczer T., Faigler S., 2016, A\&A, 589, A75

Pepe F., Mayor M., Galland F., Naef D., Queloz D., Santos N. C., Udry S., Burnet M., 2002, A\&A, 388, 632

Pepper J., et al., 2007, PASP, 119, 923

Pollacco D. L., et al., 2006, PASP, 118, 1407

Queloz D., Eggenberger A., Mayor M., Perrier C., Beuzit J. L., Naef D., Sivan J. P., Udry S., 2000, A\&A, 359, L13

Queloz D., et al., 2001, A\&A, 379, 279

Rasio F. A., Tout C. A., Lubow S. H., Livio M., 1996, ApJ, 470, 1187

Schwarz 1978, Annals of Statistics, 6, 461

Scuflaire R., Théado S., Montalbán J., Miglio A., Bourge P.-O., Godart M., Thoul A., Noels A., 2008, Ap\&SS, 316, 83

Seager S., Sasselov D. D., 2000, ApJ, 537, 916

Seager S., Richardson L. J., Hansen B. M. S., Menou K., Cho J. Y.-K., Deming D., 2005, ApJ, 632, 1122

Sedaghati E., et al., 2017, Nature, 549, 238

Sestito P., Randich S., 2005, A\&A, 442, 615

Sing D. K., et al., 2011, A\&A, 527, A73

Sing D. K., et al., 2016, Nature, 529, 59

Stassun K. G., Torres G., 2018, preprint, (arXiv:1805.03526)

Szabó G. M., Kiss L. L., 2011, ApJ, 727, L44

Talens G. J. J., Spronck J. F. P., Lesage A.-L., Otten G. P. P. L., Stuik R., Pollacco D., Snellen I. A. G., 2017, A\&A, 601, A11

Triaud A. H. M. J., 2017, The Rossiter-McLaughlin Effect in Exoplanet Research. p. 2, doi:10.1007/978-3-319-30648-3_2-1

Weiss A., Schlattl H., 2008, Ap\&SS, 316, 99

Wheatley P. J., et al., 2018, MNRAS, 475, 4476

Winn J. N., et al., 2008, ApJ, 683, 1076

This paper has been typeset from a $\mathrm{TEX}_{\mathrm{E}}$ /LATEX file prepared by the author. 